\title{
Drones Optimized Therapy System(DrOTS): Use of Drones for Tuberculosis Diagnosis in Nepal
}

\author{
Uttam Pudasaini
}

Nepal Flying Labs-Kathmandu, Nepal

\begin{abstract}
Over $50 \%$ of the total Nepalese population lives in hilly and mountainous areas with extremely poor transportation and access to health care facilities. With advanced health centers concentrated only in urban areas, and diagnostic laboratories not being present in most primary healthcare facilities, majority of people are forced to have to walk by foot, in an average 6-8 hours, to access proper healthcare facilities. Drone Optimized Therapy System, (DrOTS) aims to improve access to healthcare access in rural villages of Nepal. The pilot phase currently involved improving the accessibility of Tuberculosis diagnostic tests by linking community health workers (CHWs) with state-of-the-art diagnostic tools (GeneXpert) via drones in two municipalities of Pyuthan district, Nepal. The drones fly from central Hospitals to remote healthcare centers and bring back sputum samples for diagnosis. The purpose of this project is to assist the Ministry of Health and Population (MoHP) and National Tuberculosis Center (NTC) by generating the data necessary to assess the suitability of the drones-based services for nationwide expansion. The aerial distance between any two points being lesser than the actual road distance, drone technology has come out as a very popular tool in transporting medical samples/medicines between health centers. Drones can be used as crucial tools to connect primary healthcare facilities to hospitals by delivering patient information such as blood, urine, sputum, stool etc. samples required for diagnosis from primary facilities to hospitals, and medicines from hospitals to patients in nearby rural locations for treatment. The project team consists of multisector experts; Public Health- Birat Nepal Medical Trust (BNMT), Drones \& Tech - WeRobotics, Nepal Flying Labs \& DroNepal, Research: The Liverpool School of Tropical Medicine, Govt. stakeholders: MoHP Nepal, the National TB Center and the District Public Health Office (DPHO Pyuthan) is supported by Stony Brook University and the Simons Foundation.
\end{abstract}

